\title{
Geometry of Self-Affine Tiles I
}

\section{Robert S. Strichartz \& YANG WANG}

\begin{abstract}
For a self-similar or self-affine tile in $\mathbb{R}^{n}$ we study the following questions: 1) What is the boundary? 2) What is the convex hull? We show that the boundary is a graph directed self-affine fractal, and in the self-similar case we give an algorithm to compute its dimension. We give necessary and sufficient conditions for the convex hull to be a polytope, and we give a description of the Gauss map of the convex hull.
\end{abstract}

\section{INTRODUCTION}

A self-affine tile is a compact set $T \subseteq \mathbb{R}^{n}$ with nonempty interior that satisfies an expansion identity

$$
A(T)=\bigcup_{d \in \mathcal{D}}(T+d)
$$

where $A$ is an expanding matrix (all eigenvalues have modulus $>1$ ) and $\mathcal{D}$ is a finite set of vectors in $\mathbb{R}^{n}$, called the digit set, that satisfies $\# \mathcal{D}=|\operatorname{det} A|$. Often it is assumed that $\mathcal{D} \subseteq \mathbb{Z}^{n}$, the integer lattice, $A \mathbb{Z}^{n} \subseteq \mathbb{Z}^{n}$ (so $A$ has integer entries), and $\mathcal{D}$ is a complete residue system for $\mathbb{Z}^{n} / A \mathbb{Z}^{n}$. There is a large literature about the ways that translates of $T$ may tile $\mathbb{R}^{n}$ (see $[\mathrm{Fa}],[\mathrm{K} 1]$, [LW2] and the references therein). In this series of papers we study the geometry of the tile itself. The two main questions we answer are the following:

1 What is the boundary of $T$ ?

2 What is the convex hull of $T$ ?

To answer the first question, we will subdivide the boundary $\partial T$ into a finite number of pieces, and show that these pieces satisfy a vector version of an expansion identity like (1.1), and so form a self-affine family of sets, or graph-directed family $([\mathrm{Fa}],[\mathrm{MW}])$. The pieces of $\partial T$ that we use are just the intersections $T_{\alpha}=T \cap(T+\alpha)$ of the tile with its lattice translates $\left(\alpha \in \mathbb{Z}^{n}\right)$, so the tiling in fact determines the subdivision. We will give an algorithm for determining when $T_{\alpha}$ is nonempty. 
The need for subdivision is apparent already in the trivial example of a square, whose boundary subdivides into 4 corners and 4 sides. As this example shows, the pieces may be of different dimensions, and they do overlap, but the overlaps are small enough not to matter. Although we do not know how to verify an open set condition in all cases, we are still able (in the case that $A$ is a similarity) to compute the dimension of the boundary in terms of the spectral radius of a matrix $M$, called the boundary substitution matrix. The computation of $M$ from $A$ and $\mathcal{D}$ is given by an algorithm. An implementation of this algorithm and some specific computations are given in Part II [KLSW].

We also consider the generalization of these results to multitile systems. One motivation for this is that it enables us to handle examples that involve similarities with different rotations, such as the Levy dragon. The dimension of the boundary of the Levy dragon was first computed in [DK] by a more complicated method, involving the spectral radius of a $752 \times 752$ matrix that required a computer assisted computation. In contrast, our method requires only the spectral radius of an $11 \times 11$ matrix. The matrix itself is computed by hand, using a geometric method, and the spectral radius is the root of an explicit polynomial of degree 9 (the algebra involved in finding the characteristic polynomial of the matrix was performed using Mathematica). The geometric method we use was suggested by some of the ideas in [DK].

It is easy to see that the convex hull of $T$ is a polytope if some power of $A$ is a multiple of the identity. We show that the converse is true if $A$ is a similarity, and in general we give necessary and sufficient conditions for the convex hull to be a polytope. In the planar case $(n=2)$ we give a rather complete algorithmic description of the convex hull, including a formula for the length of the perimeter. The method we use is based on the description of $T$ as infinite digit expansion

$$
T=\left\{x=\sum_{k=1}^{\infty} A^{-k} d_{k}: d_{k} \in \mathcal{D}\right\}
$$

Given any unit vector $u$, to maximize $x \cdot u$ as $x$ varies in $T$, it is only necessary to maximize $A^{-k} d_{k} \cdot u$ as $d_{k}$ varies over $\mathcal{D}$, independently for each $k$. When there is a unique maximizer for every $k$ we obtain an extreme point of the convex hull; on the other hand, when there is a tie, the boundary of the convex hull contains a line segment. In Part II we show that the set of extreme points of the convex hull is a set of dimension zero.

The problem of determining the convex hull is of interest for the attractor of an arbitrary IFS. Our methods are very dependent on the representation (1.2), and so cannot say anything about examples involving mappings with different linear parts. However, we do not need any specific assumptions about the set $\mathcal{D}$ for the results about convex hulls. 
Acknowledgments. This research was done while the second author was a visitor in the Mathematics Department at Cornell University. We are grateful to Robert Connelly for suggesting the elegant proof of Lemma 4.3. This version of the paper is substantially revised from the first version, taking into account the suggestions from the anonymous referee and other new information, most notably the preprint of Veerman [Ve]. Before our work, Veerman had studied the Hausdorff dimension of the boundaries of self-affine tiles and obtained similar results (we shall elaborate more on this in $\S 2$ ). We retain our proofs here mainly because our method has a very clear geometric interpretation that can be useful in studying other problems. This is illustrated in obtaining the Hausdorff dimension of the boundary of the Levy Dragon in $\S 3$. In contrast, [Ve] used quite different methods that rely more on analysis. As it turns out, the substitution matrix of the boundary that we use to compute the dimension is essentially identical to the contact matrix introduced by Gröchenig and Haas [GH] to study the measure of self-affine tiles. After we completed this paper, we have received the preprint $[\mathrm{DKV}]$ which has also established similar result on the dimensions of boundaries of self-similar tiles. The methods used in [DKV] are similar to those in $[\mathrm{Ve}]$ and are rather different from ours.

\section{BOUNDARIES OF TILES}

In this section we assume that the tile $T$ satisfies

$$
A(T)=\bigcup_{d \in \mathcal{D}}(T+d)
$$

for an expanding integer matrix $A$ with $\mathcal{D} \subset \mathbb{Z}^{n}$, and that the $\mathbb{Z}^{n}$ translates of $T$ tile $\mathbb{R}^{n}$. This implies that $\mathcal{D}$ is a complete set of residues for $\mathbb{Z}^{n} / A \mathbb{Z}^{n}$ ([LW1]). If we just assume that $\mathcal{D}$ is a complete set of residues for $\mathbb{Z}^{n} / A \mathbb{Z}^{n}$, then some lattice translates of $T$ will tile $\mathbb{R}^{n}$, but it doesn't have to be $\mathbb{Z}^{n}$ ([B], [LW3]). For some results we assume that $A$ is conjugate to a similarity.

We now describe an algorithm for finding the dimension of the boundary of $T$, under the additional assumption that $A$ is conjugate to a similarity. Denote $T_{\alpha}=T \cap(T+\alpha)$ for any $\alpha \in \mathbb{Z}^{n}$ and let

$$
\mathcal{F}=\left\{\alpha \in \mathbb{Z}^{n}: \alpha \neq 0, T_{\alpha} \neq \varnothing\right\} .
$$

Then $A\left(T_{\alpha}\right)=(T+\mathcal{D}) \cap(T+\mathcal{D}+A \alpha)$, hence

$$
A\left(T_{\alpha}\right)=\bigcup_{d, d^{\prime} \in \mathcal{D}}\left(T_{A \alpha+d^{\prime}-d}+d\right) .
$$

Of course we wish to discard those terms for which $T_{A \alpha+d^{\prime}-d}$ are empty. Thus, for every $\alpha$ and $\beta$ in $\mathcal{F}$, we let $C(\alpha, \beta)$ denote the set of pairs $\left(d, d^{\prime}\right) \in \mathcal{D} \times \mathcal{D}$ 
such that

$$
\beta=A \alpha+d^{\prime}-d
$$

Then we can rewrite $(2.2)$ as

$$
T_{\alpha}=\bigcup_{\beta} \bigcup_{\left(d, d^{\prime}\right) \in C(\alpha, \beta)}\left(A^{-1} T_{\beta}+A^{-1} d\right) .
$$

All the sets on the right side of (2.4) are nonempty compact sets. Thus (2.4) is a graph-directed construction for the self-similar family of sets $\left\{T_{\alpha}: \alpha \in \mathcal{F}\right\}$. It is known $[\mathrm{Fa}]$ that (2.4) uniquely determines the sets (provided we assume that they are nonempty compact sets) and it provides an iterative procedure for approximating the sets, starting with any initial choices of nonempty compact sets. Usually one would want to have an open set condition on the unions in (2.4) in order to compute the dimension of the sets. The Hausdorff dimension of the boundary of $T$ can be determined if the graph-directed IFS (2.4) satisfies the open set condition, but this is not clear; nevertheless we find a way to compute the dimension.

Key to our study is the substitution matrix $M=\left(M_{\alpha \beta}\right)$ of the boundary, where $\alpha, \beta \in \mathcal{F}$ and $M_{\alpha \beta}=\# C(\alpha, \beta) .{ }^{1}$ We write $\rho(M)$ for the spectral radius of $M$ and $r$ for the expansion ratio of $A$. The formula for the dimension of the boundary is simply

$$
\log \rho(M) / \log r
$$

which is the same value that holds under the open set condition.

Remark. Since $T_{-\alpha}=T_{\alpha}-\alpha$ we can reduce the size of the matrix $M$ by half if we choose one of each pair $\pm \alpha$ in $\mathcal{F}$. We do this when convenient in calculations, but it is easier to write the proofs without this simplification.

Lemma 2.1. If $A$ is conjugate to a similarity of expansion ratio $r$, then the box dimension of the boundary of $T$ satisfies $\overline{\operatorname{dim}}_{B}(\partial T) \leq \log \rho(M) / \log r$.

Proof. Without loss of generality we assume that $A$ is a similarity. We need to use iterates of (2.4). So for any $N \geq 1$, there exist sets $\mathcal{E}_{N, \alpha, \beta}$ (subsets of $\mathbb{Z}^{n}$ ) such that

$$
T_{\alpha}=\bigcup_{\beta \in \mathcal{F}} A^{-N}\left(T_{\beta}+\mathcal{E}_{N, \alpha, \beta}\right),
$$

${ }^{1}$ This matrix is essentially identical to the contact matrix introduced in [GH], used to determine the measure of a self-affine tile. 
where $\mathcal{E}_{1, \alpha, \beta}=\left\{d:\left(d, d^{\prime}\right) \in C(\alpha, \beta)\right\}$ and

$$
\mathcal{E}_{N+1, \alpha, \beta}=\bigcup_{\gamma \in \mathcal{F}}\left(A \mathcal{E}_{N, \alpha, \gamma}+\mathcal{E}_{1, \gamma, \beta}\right) .
$$

We claim that all the elements on the right side of (2.7) are disjoint, which implies that the matrix $\left(\# \mathcal{E}_{N, \alpha, \beta}\right)$ is just $M^{N}$ for any $N$.

To prove the claim we make use of the fact that $\mathcal{E}_{1, \alpha, \beta}$ is a subset of $\mathcal{D}$, and $\mathcal{D}$ is a complete residue system for $\mathbb{Z}^{n} / A \mathbb{Z}^{n}$. Now suppose $A x+y=A x^{\prime}+y^{\prime}$ for some $x \in \mathcal{E}_{N, \alpha, \gamma}, y \in \mathcal{E}_{1, \gamma, \beta}$ and $x^{\prime} \in \mathcal{E}_{N, \alpha, \gamma^{\prime}}, y \in \mathcal{E}_{1, \gamma^{\prime}, \beta}$. This can only happen if $y=y^{\prime}$ and $x=x^{\prime}$. Now $y=y^{\prime}$ implies $\gamma^{\prime}=\gamma$, for by (2.3) we have both $\beta=A \gamma+d-d^{\prime}$ and $\beta=A \gamma^{\prime}+d-d^{\prime \prime}$, hence $A\left(\gamma-\gamma^{\prime}\right)=d^{\prime}-d^{\prime \prime}$. This proves $\gamma=\gamma^{\prime}$, so $A x+y$ and $A x^{\prime}+y^{\prime}$ are identical elements in (2.7).

To establish the upper bound for $\overline{\operatorname{dim}}_{B}(\partial T)$ it is sufficient to do so for each $\overline{\operatorname{dim}}_{B}\left(T_{\alpha}\right)$. Let $C_{\alpha}(\varepsilon)$ denote the smallest number of cubes of side length $\varepsilon$ needed to cover $T_{\alpha}$. We choose $\delta$ so that any $T_{\alpha}$ may be covered by a single cube of size $\delta$. Then from (2.6) we obtain

$$
C_{\alpha}\left(r^{-N} \delta\right) \leq \sum_{\beta} \# \mathcal{E}_{N, \alpha, \beta}=\sum_{\beta}\left(M^{N}\right)_{\alpha, \beta},
$$

hence

as $N \rightarrow \infty$. This yields

$$
\frac{\log C_{\alpha}\left(r^{-N} \delta\right)}{\log \delta^{-1}+N \log r} \leq \frac{\log \sum_{\beta}\left(M^{N}\right)_{\alpha, \beta}}{N \log r} \rightarrow \frac{\log \rho(M)}{\log r}
$$

$$
\overline{\operatorname{dim}}_{B}\left(T_{\alpha}\right) \leq \log \rho(M) / \log r .
$$

Remark. We obtain the same conclusion under slightly weaker hypotheses on $A$ : all eigenvalues of $A$ have the same modulus $r$. In that case we have estimates $\left\|A^{N} x\right\| \leq c r^{N}(1+N)^{b}\|x\|$ for constants $c$ and $b$ independent of $N$, and similar estimates from below, and so the same proof will work.

Theorem 2.2. Let $T$ be a self-affine tile satisfying $A(T)=T+\mathcal{D}$ for some expanding matrix $A \in M_{n}(\mathbb{Z})$ and digit set $\mathcal{D} \subset \mathbb{Z}^{n}$ that is a residue system of $\mathbb{Z}^{n} / A \mathbb{Z}^{n}$. Suppose that $A$ is conjugate to a similarity with expansion ration $r$, and the $\mathbb{Z}^{n}$ translates of $T$ tile $\mathbb{R}^{n}$. Then $\operatorname{dim}_{H}(\partial T)=\operatorname{dim}_{B}(\partial T)=\log \rho(M) / \log r$. Furthermore, $\mathcal{H}^{s}(\partial T)>0$ for $s=\operatorname{dim}_{H}(\partial T)$.

Proof. Without loss of generality we assume $A$ is a similarity. We write (2.6) for $N=1$ as

$$
A\left(T_{\alpha}\right)=\bigcup_{\beta \in \mathcal{F}}\left(T_{\beta}+\mathcal{E}_{\alpha, \beta}\right),
$$


where $\mathcal{E}_{\alpha, \beta}$ stands for $\mathcal{E}_{1, \alpha, \beta}, \mathcal{E}_{\alpha, \beta} \subseteq \mathcal{D}$. The proof of Lemma 2.1 already implies that for a fixed $\beta$ the sets $\left\{\mathcal{E}_{\alpha, \beta}: \alpha \in \mathcal{F}\right\}$ are disjoint. Hence $\bigcup_{\alpha} \mathcal{E}_{\alpha, \beta} \subseteq \mathcal{D}$.

Now we may assume that

$$
M=\left(\begin{array}{cccc}
M_{1} & * & \cdots & * \\
0 & M_{2} & \cdots & * \\
\vdots & \vdots & \ddots & \vdots \\
0 & 0 & \cdots & M_{r}
\end{array}\right)
$$

where each $M_{i}$ is irreducible with dimension $m_{i} \times m_{i}$. This can always be achieved by a suitable permutation of indices. Since $\max \left(\rho\left(M_{i}\right)\right)=\rho(M)$, we may assume without loss of generality that $\rho\left(M_{1}\right)=\rho(M)$.

Suppose that $M_{1}$ corresponds to the indices $\{\alpha\}=\widehat{\mathcal{F}} \subseteq \mathcal{F}$. We consider the following vector (graph-directed) IFS

$$
A\left(\widehat{T}_{\alpha}\right)=\bigcup_{\beta \in \widehat{\mathcal{F}}}\left(\widehat{T}_{\beta}+\mathcal{E}_{\alpha, \beta}\right), \quad \alpha \in \widehat{\mathcal{F}}
$$

Clearly, $\widehat{T}_{\alpha} \subseteq T_{\alpha}$. Since $\bigcup_{\alpha} \mathcal{E}_{\alpha, \beta} \subseteq \mathcal{D}$, we can augment $\mathcal{E}_{\alpha, \beta}$ to $\widetilde{\mathcal{E}}_{\alpha, \beta}$ so that $\bigcup_{\alpha} \widetilde{\mathcal{E}}_{\alpha, \beta}=\mathcal{D}$. Because $M_{1}$ is irreducible, the graph-directed IFS

$$
A\left(\widetilde{T}_{\alpha}\right)=\bigcup_{\beta \in \widehat{\mathcal{F}}}\left(\widetilde{T}_{\beta}+\widetilde{\mathcal{E}}_{\alpha, \beta}\right), \quad \alpha \in \widehat{\mathcal{F}}
$$

gives self-affine multitiles $\left\{\widetilde{T}_{\alpha}\right\}$ such that $\widetilde{T}_{\alpha}^{o} \neq \varnothing$ and $\widetilde{T}_{\alpha}$ is the closure of its interior, see $[\mathrm{FW}]$. Hence

$$
A\left(\widetilde{T}_{\alpha}^{o}\right) \supseteq \bigcup_{\beta \in \widehat{\mathcal{F}}}\left(\widetilde{T}_{\beta}^{o}+\widetilde{\mathcal{E}}_{\alpha, \beta}\right) \supseteq \bigcup_{\beta \in \widehat{\mathcal{F}}}\left(\widetilde{T}_{\beta}^{o}+\widehat{\mathcal{E}}_{\alpha, \beta}\right) .
$$

The unions on the right of (2.9) are disjoint. Thus $\left\{\widetilde{T}_{\alpha}^{o}\right\}$ yields open set condition for $\left\{\widehat{T}_{\alpha}\right\}$, and hence $([\mathrm{MW}])$

$$
\operatorname{dim}_{H}\left(\widehat{T}_{\alpha}\right)=s=\frac{\log \rho\left(M_{1}\right)}{\log r}, \quad \mathcal{H}^{s}\left(\widehat{T}_{\alpha}\right)>0
$$

Note that each $\widehat{T}_{\alpha} \subseteq \partial T$, and $\overline{\operatorname{dim}_{B}}(\partial T) \leq s$. The theorem follows.

Corollary 2.3. Under the hypotheses of Theorem 2.2, the dimension of the boundary of $T$ is always strictly less than $n$. 
Proof. If the dimension of $\partial T$ were equal to $n$, then by the previous theorem it would have to have positive Lebesgue measure. But this contradicts the known fact ([LW2]) that $\partial T$ must have Lebesgue measure zero.

We consider next the question of whether or not the boundary of $T$ has finite Hausdorff measure in its dimension. The answer is given in terms of the matrix $M$. The essential condition we need is that $\rho(M)^{-k} M^{k}$ be uniformly bounded as $k \rightarrow \infty$. It is easy to see by linear algebra that this is equivalent to the condition that the generalized eigenspace associated to $\rho(M)$ be equal to the eigenspace for $\rho(M)$.

Theorem 2.4. Under the hypotheses of Theorem 2.2, the boundary of $T$ has finite Hausdorff measure provided that the generalized eigenspace associated to the eigenvalue $\rho(M)$ of $M$ coincides with the eigenspace.

Proof. Our hypothesis implies that $\rho(M)^{-k} M^{k}$ is uniformly bounded. Then (2.6) gives coverings of $\partial T$ that show that the Hausdorff measure is finite.

The converse of the theorem is also true, but we will not give the proof here. This is perhaps a moot point, since Theorem 3.2 in [Fa] would imply directly that the Hausdorff measure is always finite. However, we have not been able to show that this result is applicable here. In all the examples we have computed in Part II [KLSW], the hypothesis of Theorem 2.4 is verified. In particular, we know of no examples with infinite Hausdorff measure.

Remark. The assumption that $T$ tiles $\mathbb{R}^{n}$ by translates of $\mathbb{Z}^{n}$ can be dropped. Without this assumption, one may remove from $M$ all irreducible components whose spectral radii are $n$, and Theorem 2.2 remains true.

Remark. A slightly different dimension formula was given in [Ve]. A simple transformation shows, however, that the two formulas are the same. In the case when the expanding matrix is not a similarity, an estimate of the dimension is given in $[\mathrm{Ve}]$. This paper establishes stronger results on the Hausdorff measure of the boundary, showing that the measure is always positive and giving necessary and sufficient conditions for it to be finite. In [Ve] some sufficient conditions were given.

Example 2.5 (Twin Dragon). Let

$$
A=\left(\begin{array}{cc}
1 & -1 \\
1 & 1
\end{array}\right)
$$


and $\mathcal{D}=\{(0,0),(1,0)\}$. Then $T$ is the well-known twin dragon tile. It is easy to see that $\mathcal{F}=\{(1,0),(-1,0),(0,1),(0,-1),(1,-1),(-1,1)\}$, and since $T_{\alpha}$ and $T_{-\alpha}$ are translates of each other, we can reduce the computation by considering only 3 intersections: $T_{(1,0)}, T_{(0,1)}$ and $T_{(-1,1)}$. We then have from (2.2) that

$$
\left\{\begin{array}{l}
A T_{(1,0)}=T_{(0,1)}+(1,0), \\
A T_{(0,1)}=T_{(0,1)} \cup T_{(-1,1)} \cup\left(T_{(-1,1)}+(1,0)\right), \\
A T_{(-1,1)}=T_{(1,0)}+(-1,0),
\end{array}\right.
$$

which gives the matrix

$$
M=\left(\begin{array}{lll}
0 & 1 & 0 \\
0 & 1 & 2 \\
1 & 0 & 0
\end{array}\right),
$$

so $\rho(M)$ is the largest root of $\lambda^{3}-\lambda^{2}-2=0, \lambda \approx 1.69562076956$ and the dimension of the boundary is $2 \log \lambda / \log 2 \approx 1.52362708621$. In this example it is easy to see that the pieces $T_{(1,0)}, T_{(0,1)}$ and $T_{(-1,1)}$ of the boundary intersect each other in single points. Figure 2.1 (a) shows the positions of the tiles $T$, $T+(1,0), T+(0,1)$ and $T+(-1,1)$, so the intersections are apparent. Figure 2.1 (b) shows the subdivision of these tiles into translates of $A^{-1} T$, which makes it possible to visualize the stated decomposition rules.

In the appendix we shall give a simple algorithm for finding the set $\mathcal{F}$. We show also that in defining the substitution matrix of the boundary one can use any index set $\mathcal{F}^{\prime}$, as long as $\mathcal{F}^{\prime} \supseteq \mathcal{F}$.

\section{The Levy Dragon}

One advantage our method has is that it actually works in the more general setting of self-similar multitiles, in which the self-affine tile $T$ is replaced by a set of prototiles $T_{1}, T_{2}, \ldots, T_{r}$ in $\mathbb{R}^{n}$, each with nonempty interior, that form a self-similar system

$$
A\left(T_{j}\right)=\bigcup_{k}\left(T_{k}+\mathcal{D}_{j k}\right)
$$

for a matrix of digit sets $\mathcal{D}_{j k} \subseteq \mathbb{Z}^{n}$. It must be understood that if $\mathcal{D}_{j k}$ is empty, then there is no contribution by translates of $T_{k}$ to the union. We will assume that the $\mathbb{Z}^{n}$ translates of $T_{k}, k=1,2, \ldots, r$, tile $\mathbb{R}^{n}$. It is proved in [FW] that under such an assumption each $\mathcal{D}_{k}=\bigcup_{j} \mathcal{D}_{j k}$ is necessarily a complete set of residues for $\mathbb{Z}^{n} / A \mathbb{Z}^{n}$ and the matrix $\left(\# \mathcal{D}_{j k}\right)$ is primitive. We are again interested in the boundaries of each of the prototiles, and so we define

$$
T(j, k, \alpha)=T_{j} \cap\left(T_{k}+\alpha\right), \quad \alpha \in \mathbb{Z}^{n}
$$




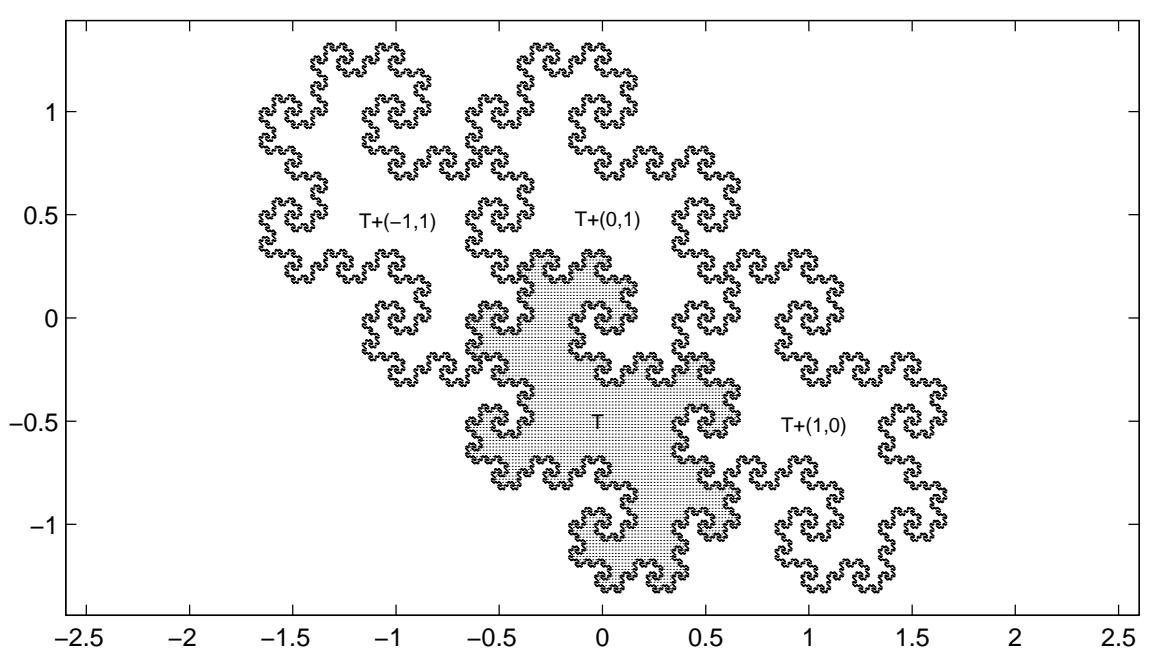

(a)

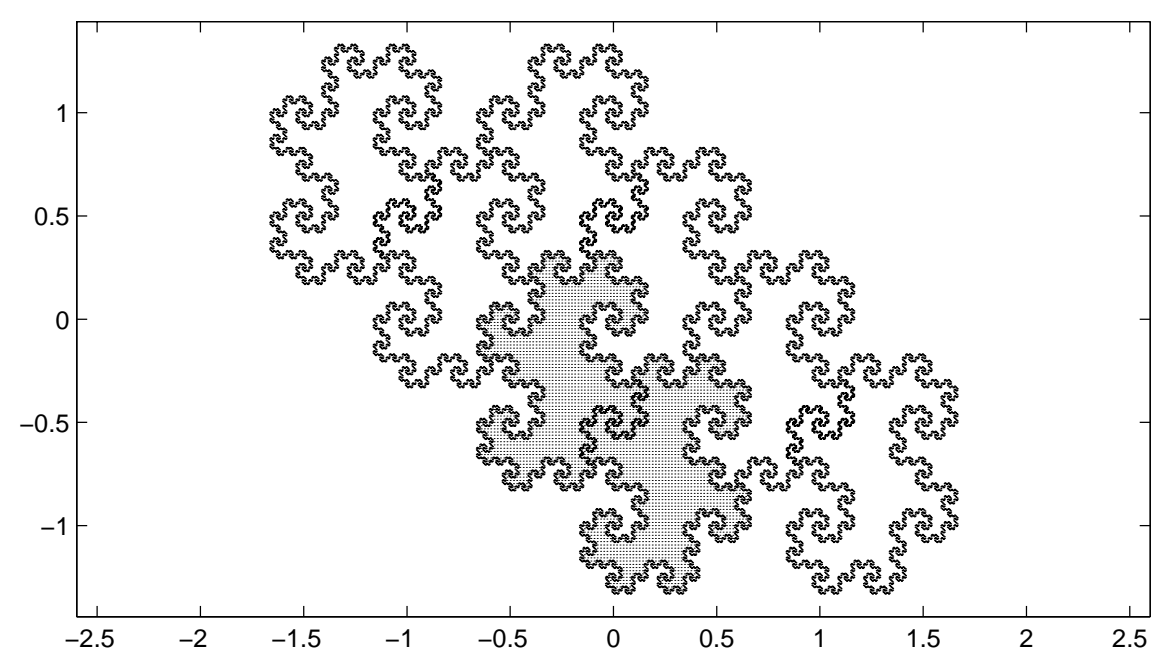

(b)

Figure 2.1. (a) The twin dragon $T$ and 3 adjacent tiles $T+$ $(1,0), T+(0,1)$ and $T+(-1,1)$. (b) The subdivision of the tiles in (a) into translates of $A^{-1} T$. 
and

$$
\mathcal{F}=\{(j, k, \alpha): T(j, k, \alpha) \neq \varphi \text { and if } j=k \text { then } \alpha \neq 0\} .
$$

A pruning algorithm analogous to Theorem A.3 allows the computation of $\mathcal{F}$. The analog of (2.2) is the following:

$$
\begin{aligned}
A T(j, k, \alpha) & =A T_{j} \cap\left(A T_{k}+A \alpha\right) \\
& =\bigcup_{\ell, m} \bigcup_{\delta_{j \ell} \in \mathcal{D}_{j \ell}} \bigcup_{\delta_{k m} \in \mathcal{D}_{k m}}\left(T_{\ell}+\delta_{j \ell}\right) \cap\left(T_{m}+\delta_{k m}+A \alpha\right) \\
& =\bigcup_{\ell, m} \bigcup_{\delta_{j \ell} \in \mathcal{D}_{j \ell}} \bigcup_{\delta_{k m} \in \mathcal{D}_{k m}}\left(T\left(\ell, m, A \alpha+\delta_{k m}-\delta_{j \ell}\right)+\delta_{j \ell}\right) .
\end{aligned}
$$

The sets $C((j, k, \alpha),(\ell, m, \beta))$ and the matrix $M$ of size $\# \mathcal{F} \times \# \mathcal{F}$ are defined by

$$
C((j, k, \alpha),(\ell, m, \beta))=\left\{\left(\delta_{k m}, \delta_{j \ell}\right) \in \mathcal{D}_{k m} \times \mathcal{D}_{j \ell}: A \alpha+\delta_{k m}-\delta_{j \ell}=\beta\right\}
$$

and

$$
M_{(j, k, \alpha),(\ell, m, \beta)}=\# C((j, k, \alpha),(\ell, m, \beta)) .
$$

The analog of (2.4) is

$$
T(j, k, \alpha)=\bigcup_{(k, m, \beta) \in \mathcal{F}} \bigcup_{C((j, k, \alpha),(\ell, m, \beta))}\left(A^{-1} T(\ell, m, \beta)+A^{-1} \delta_{j \ell}\right) .
$$

With these modifications, Theorem 2.2, Corollary 2.3 and Theorem 2.4 continue to hold, and the proofs are essentially the same. One important application is for certain tilings with variable rotations, in which all the prototiles are just rotated copies of a single one. We illustrate this with the next example.

The Levy Dragon. There are 4 prototiles $T_{0}, T_{1}, T_{2}, T_{3}$, but in fact $T_{j}$ is a rotation of $T_{0}$ through angle $j \pi / 2$. The matrix $A=\left(\begin{array}{cc}1 & -1 \\ 1 & 1\end{array}\right)$ as with the twin dragon, and the expansion identities are

$$
\left\{\begin{array}{l}
A\left(T_{0}\right)=T_{2} \cup T_{3}, \\
A\left(T_{1}\right)=\left(T_{1}+(-1,1)\right) \cup T_{2}, \\
A\left(T_{2}\right)=\left(T_{2}+(-1,0)\right) \cup\left(T_{3}+(0,1)\right), \\
A\left(T_{3}\right)=\left(T_{0}+(-1,1)\right) \cup T_{3} .
\end{array}\right.
$$

The usual description involves an IFS of 2 similarities involving rotations of $\pm \pi / 4$ for the single tile $T_{0}$, but it is easily seen to be equivalent to the above system. As is explained in [DK], the tiling by $\mathbb{Z}^{n}$ translates of $T_{0}, T_{1}, T_{2}, T_{3}$ can be easily visualized by considering the corresponding tiling by right triangles obtained by drawing both diagonals of each square of the unit square tiling. Denote by $\widetilde{T}_{0}$, 


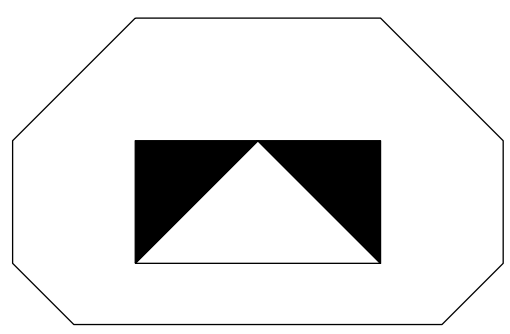

Figure 3.1. The relative position and size of the triangle $\widetilde{T}_{0}$, the 2 smaller triangles given by the symbolic transformation law, and the convex hull of $T_{0}$ (an octagon).

$\widetilde{T}_{1}, \widetilde{T}_{2}, \widetilde{T}_{3}$ the triangles composing the unit square. If we apply $A^{-1}$ to both sides of (3.5) and replace each $T_{j}$ by $\widetilde{T}_{j}$, we no longer get equalities, but we can interpret the results as a symbolic transformation law for passing from the $\mathbb{Z}^{n}$ tiling by $T_{0}, T_{1}, T_{2}, T_{3}$ to the $A^{-1} \mathbb{Z}^{n}$ tiling by $A^{-1} T_{0}, A^{-1} T_{1}, A^{-1} T_{2}, A^{-1} T_{3}$. The rule is simply that each triangle gets replaced by 2 triangles (contracted by the factor $2^{-1 / 2}$ ) whose hypotenuses lie on the equal sides of the large triangle, and which lie on the outside of the large triangle. This is shown in Figure 3.1, which also shows the relative position of the convex hull of $T_{0}$ and the triangle $\widetilde{T}_{0}$.

We will use a geometric method rather than the algebraic method discussed above to find $\mathcal{F}$ and the decomposition (3.4) for this example. First we observe that tiles in the tiling can intersect only if their convex hulls intersect. It turns out in this example that the converse is also true. Also, if the convex hulls intersect in a line or a point, the intersection of the tiles is trivial for the purposes of computing dimension. It turns out that there is another trivial intersection (a point) for which the convex hulls have nontrivial intersection. After taking into account symmetries, we are left with 11 types of nontrivial intersections. In Figure 3.2 we show the corresponding triangles, labeled from 1 to 11 (multiple use of the same number corresponds to isometric intersections). There is no particular significance to the choice of number labels. We use $P$ and $L$ to label point and subset of line intersections, and $P^{*}$ labels the nonobvious point intersection. In Figure 3.3 we analyze each of these 11 types of nontrivial intersections and the nonobvious point intersection under the symbolic transformation law. Each diagram shows 2 triangles in the relative positions of triangle $\widetilde{T}_{0}$ and one other triangle, and then the 4 smaller triangles which result from the symbolic transformation. The small triangles are then examined for their relative position (on the smaller scale), and the result is recorded in the transformation rule beneath the diagram. There are 4 pairs of small triangles to be examined, and some may 


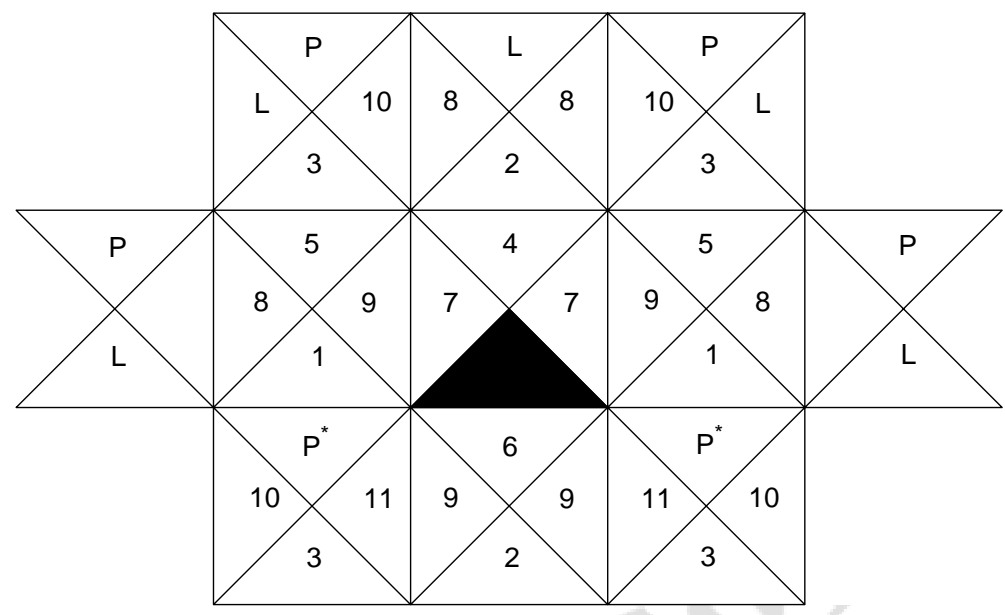

FIGURE 3.2. All triangles such that the convex hull of the associated tile meets the convex hull of $T_{0}$. The triangle $\widetilde{T}_{0}$ is black, the numbers 1 through 11 are used to label nontrivial intersections, with the same number used for isometric intersections. The label $L$ means that the intersection of the convex hulls is a line segment, and $P$ means it is a point. $P^{*}$ means the intersection of the tiles is a point, even though the convex hulls intersect nontrivially.

result in empty intersections. For example, the first diagram shows that the intersection of $T_{0}$ and $T_{0}+(1,0)$ (which is labelled (1)), breaks up into 2 copies of (3) and one copy of (7). The choice of corresponding small triangles produces the 2 copies of (3), and the choice of adjacent small triangles produces a copy of (7), while the 2 distant small triangles produce an empty intersection. The symbol $(1) \rightarrow(3) \cup(3) \cup(7)$ summarizes these observations. The last diagram explains why $P^{*}$ is just a point, since it decomposes into just one $P^{*}$. In Figure 3.4 we display the actual intersections of the tiles corresponding to the first six diagrams in Figure 3.3.

The geometric computations done in Figure 3.3 provide all the relevant information in (3.4) (we have not bothered to compute the translation vectors $A^{-1} \delta_{j \ell}$, but that could be done if desired). In particular we can read off the matrix $M$, or rather the reduced form where we discard the contributions for $P$ and $L$ intersections. (If we took these into account we would end up with a larger matrix with the same spectral radius.) We have 


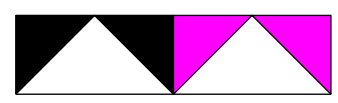

$(1) \rightarrow(3) \cup(3) \cup(7)$

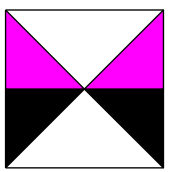

$(4) \rightarrow(7) \cup(7) \cup\left(P^{*}\right) \cup\left(P^{*}\right)$

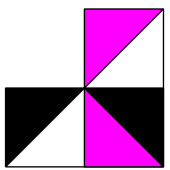

$(7) \rightarrow(1) \cup(6) \cup(9) \cup(9)$
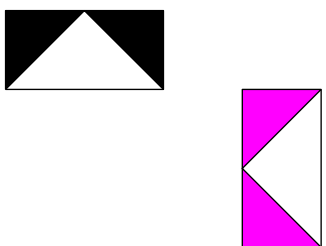

$(10) \rightarrow(8) \cup(P)$
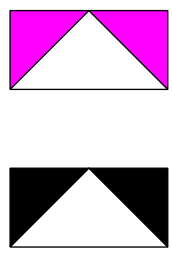

$(2) \rightarrow(3) \cup(3)$

$(3) \rightarrow(10) \cup(\mathrm{L})$

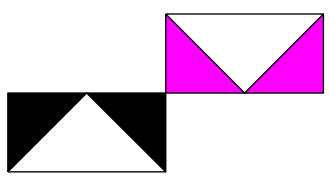

$(5) \rightarrow(4) \cup(10) \cup(10)$
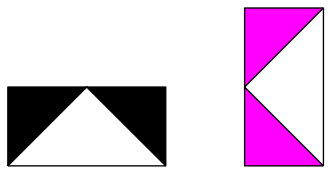

$(8) \rightarrow(5) \cup(8)$

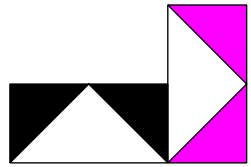

$(9) \rightarrow(2) \cup(9) \cup(L)$
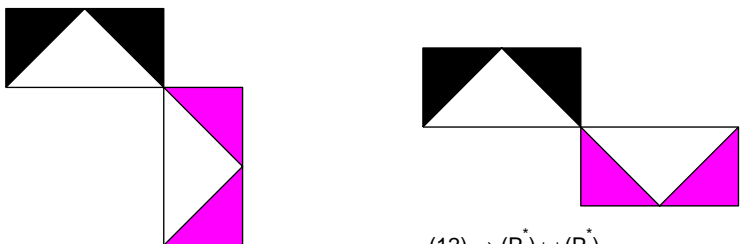

$(12) \rightarrow\left(P^{*}\right) \cup\left(P^{*}\right)$

Figure 3.3. The diagrams analyzing the 11 nontrivial intersections and the $P^{*}$ intersection. Each diagram shows 2 white triangles in the given position and the 4 smaller triangles obtained under the symbolic transformation law. The small triangles arising from triangle $\widetilde{T}_{0}$ are black, and those arising from the other white triangle are grey. (Note that in (7) there are black and grey triangles overlapping the white triangles.) Below each diagram is a summary of the nonempty intersection types of the black and grey triangles. 


$$
M=\left(\begin{array}{lllllllllll}
0 & 0 & 2 & 0 & 0 & 0 & 1 & 0 & 0 & 0 & 0 \\
0 & 0 & 2 & 0 & 0 & 0 & 0 & 0 & 0 & 0 & 0 \\
0 & 0 & 0 & 0 & 0 & 0 & 0 & 0 & 0 & 1 & 0 \\
0 & 0 & 0 & 0 & 0 & 0 & 2 & 0 & 0 & 0 & 0 \\
0 & 0 & 0 & 1 & 0 & 0 & 0 & 0 & 0 & 2 & 0 \\
0 & 0 & 0 & 0 & 0 & 0 & 0 & 0 & 0 & 0 & 2 \\
1 & 0 & 0 & 0 & 0 & 1 & 0 & 0 & 2 & 0 & 0 \\
0 & 0 & 0 & 0 & 1 & 0 & 0 & 1 & 0 & 0 & 0 \\
0 & 1 & 0 & 0 & 0 & 0 & 0 & 0 & 1 & 0 & 0 \\
0 & 0 & 0 & 0 & 0 & 0 & 0 & 1 & 0 & 0 & 0 \\
1 & 0 & 0 & 0 & 0 & 0 & 0 & 0 & 0 & 0 & 0
\end{array}\right)
$$

The characteristic polynomial factors into the trivial factors $\lambda(\lambda+1)$ and the 9th degree polynomial

$$
\lambda^{9}-3 \lambda^{8}+3 \lambda^{7}-3 \lambda^{6}+2 \lambda^{5}+4 \lambda^{4}-8 \lambda^{3}+8 \lambda^{2}-16 \lambda+8 .
$$

Thus $\rho(M)$ is the largest root of (3.6) which may be computed by Newton's method to any desired accuracy,

$$
\rho(M) \approx 1.954776399102463907266 \ldots
$$

and so the dimension of the boundary is

$$
\log \rho(M) / \log \sqrt{2} \approx 1.934007182988290978 \ldots .
$$

This computation agrees with [DK]. The matrix $M$ is irreducible, so Theorem 2.4 applies. Each of the 11 types of intersections have finite nonzero Hausdorff measure, and the relative Hausdorff measures are determined by the positive eigenvector associated with $\rho(M)$ (also directly from the relations in Figure $3.3)$.

\section{Convex hulls}

In this section we drop the assumption that $T$ is a tile. We assume only that $T$ satisfies (1.1) for some expanding matrix $A$ and some finite set $\mathcal{D}$. Thus $T$ is the attractor of an affine IFS where all the mappings have the same linear part.

Let $K$ denote the convex hull of $T$, and let $E$ denote the extreme points of $K$. The first question that arises is: when is $E$ a finite set? In that case $K$ is a polytope. It is well-known that in the more general context of self-similar fractals defined by a linear IFS, if the linear part of each mapping is a positive multiple of the identity, then the convex hull of the fractal is just the convex hull of the fixed points of the mappings. In our context this means that if some power of $A$ is a positive multiple of the identity, then $E$ is finite, since we can 


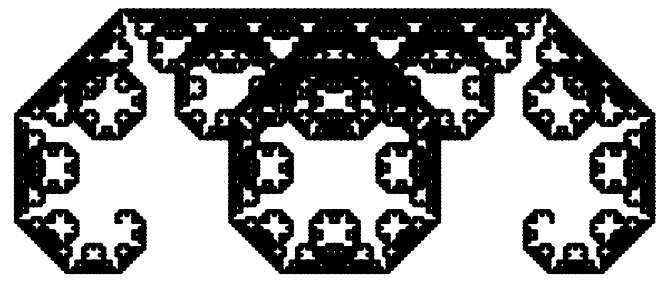

(1)

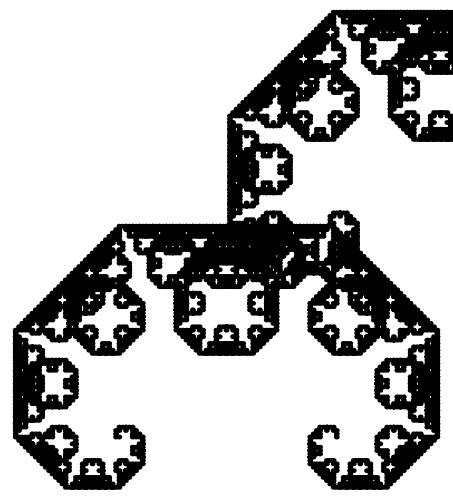

(3)

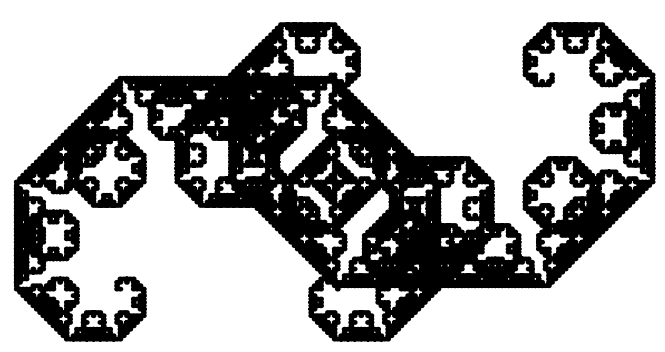

(5)

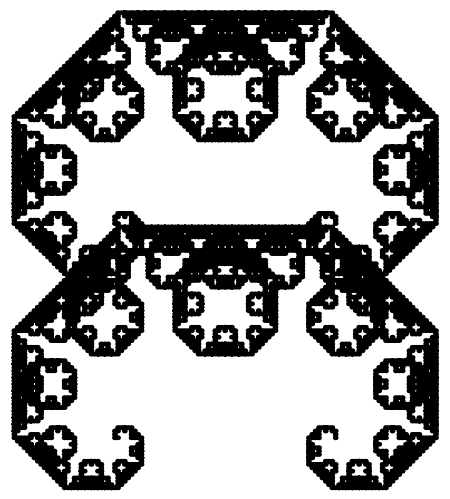

(2)

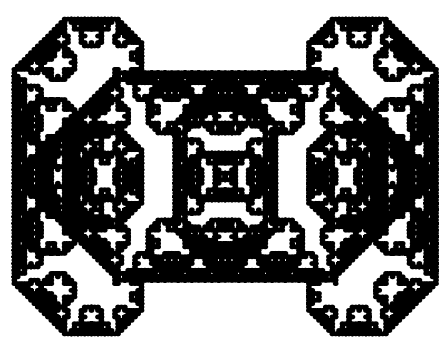

(4)

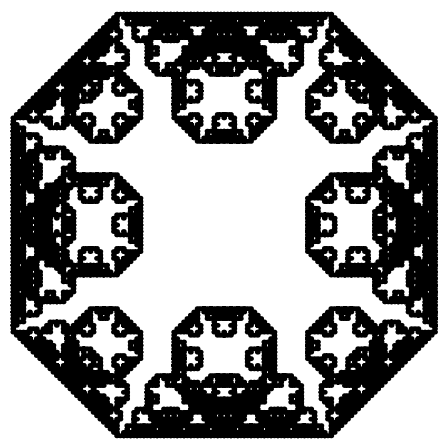

(6)

Figure 3.4. The actual intersections of tiles associated to the first 6 of the diagrams in Figure 3.3. 


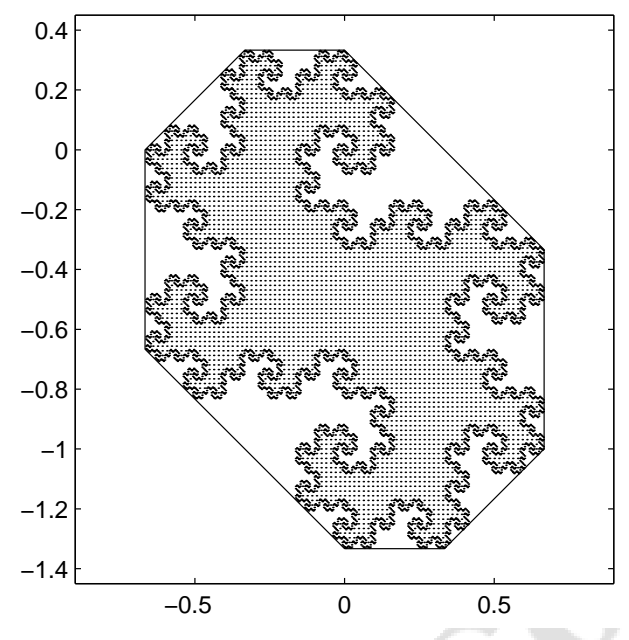

Figure 4.1. The twin dragon and its convex hull, an octagon.

always represent $T$ as the self-similar fractal represented by the IFS obtained by iterating the original one,

$$
A^{-1}\left(x+d_{j}\right), \quad d_{j} \in \mathcal{D} .
$$

In the case of the twin dragon, $A$ involves a $45^{\circ}$ rotation so $A^{8}$ is the smallest power that is a positive multiple of the identity. The 8 times iterated IFS contains $2^{8}=256$ mappings, so this argument only yields the estimate $\# E \leq 256$. In fact $\# E=8$, as can be seen from Figure 4.1. The methods of this section will also reveal this. However, the methods do not seem to work for multitiles. Nevertheless, the boundary of the convex hull of the Levy dragon is also an octagon.

The main idea of this section is to study the Gauss map of $K$. To every given unit vector $u$, we ask which points $x$ in $T$ maximize $x \cdot u$. Since every $x$ in $T$ has a representation

$$
x=\sum_{k=1}^{\infty} A^{-k} d_{k} \quad \text { for } d_{k} \in \mathcal{D},
$$

and the digits $d_{k}$ may be chosen independently, it is clear that $x$ maximizes $x \cdot u$ if and only if $\left(A^{-k} d_{k}\right) \cdot u$ maximizes $\left(A^{-k} d\right) \cdot u$ as $d$ varies over $\mathcal{D}$. In particular, if there is a unique maximizer for each $k$, then there is a unique maximizer for $x \cdot u$, and this is an extreme point. On the other hand, if $x \cdot u$ does not have a unique maximizer, then there must be a tie for the maximum of $\left(A^{-k} d\right) \cdot u=d \cdot\left(A^{-k}\right)^{*} u$, so $\left(A^{-k}\right)^{*} u$ must be perpendicular to a line segment joining two points of $\mathcal{D}$. For 
multitiles we have a similar representation for each $x$ in $T_{j}$, except now the digits $d_{k}$ must be chosen from $\mathcal{D}_{j_{k-1} j_{k}}$ for some sequence $j_{0}, j_{1}, \ldots$ with $j_{0}=j$ and $\mathcal{D}_{j_{k-1} j_{k}}$ nonempty for all $k$. Thus the sequence of digits $d_{k}$ may not be chosen independently, so nothing we say here applies to this case.

In fact we do not have to consider all of $\mathcal{D}$, but only the subset $\mathcal{D}_{0}$ of extreme points of the polytope $P$ equal to the convex hull of $\mathcal{D}$. We also note that the set of fixed-points of the IFS is equal to $(A-I)^{-1} \mathcal{D}$, so the convex hull of the fixed points $P^{\prime}$ is equal to $(A-I)^{-1} P$, and has $(A-I)^{-1} \mathcal{D}_{0}$ as its extreme points.

For simplicity we consider first the case of planar tiles, $n=2$. Then $P$ is a polygon (perhaps degenerating to a line segment). We denote by $n_{j}$ all the outward unit normal vectors to the sides $e_{j}$ of $P$. (If $P$ is a line segment, we count both directions as separate sides).

Theorem 4.1. Let $T$ be a nonempty compact set satisfying $A(T)=T+\mathcal{D}$ for an expanding matrix $A \in M_{2}(\mathbb{R})$ and a finite digit set $\mathcal{D} \subset \mathbb{R}^{2}$. Let $\left\{n_{j}\right\}$ be the outward unit normal vectors of the sides of the convex hull of $\mathcal{D}$. Then the convex hull $K$ of $T$ is a polygon if and only if every $n_{j}$ is an eigenvector of $\left(A^{*}\right)^{k}$ for some $k$. In particular, if $A$ is a similarity, then $K$ is a polygon if and only if there exists $k$ such that $A^{k}$ is a multiple of the identity.

Proof. The condition that each $n_{j}$ is an eigenvector of some $\left(A^{*}\right)^{k}$ is easily seen to be equivalent to the statement that the set of all directions of the vectors $\left(A^{*}\right)^{k} n_{j}$ is finite. Now if $u$ is a unit vector in the direction of $\left(A^{*}\right)^{k} n_{j}$, then the set of points $x$ in $T$ maximizing $x \cdot u$ contains at least 2 points, so the boundary of $K$ contains a line segment perpendicular to $u$. If there are an infinite number of such directions then $K$ is not a polygon. On the other hand, if there are only a finite number, say $u_{1}, u_{2}, \ldots, u_{m}$ in counterclockwise order, we claim $K$ is the polygon whose vertices are the points in $T$ that simultaneously maximize $x \cdot u_{j}$ and $x \cdot u_{j+1}$ (identify $u_{1}=u_{m}$ ). To see that such points exist consider the digits that maximize $\left(A^{*}\right)^{k} u_{j} \cdot d$ and $\left(A^{*}\right)^{k} u_{j+1} \cdot d$, for any fixed $k$. They must be the same, for if not there would be a direction $v$ in between with a tie, contradicting the choice of the $u_{j}$.

In the case of the twin dragon, the convex hull $P$ of $\mathcal{D}$ is a line segment and there are only two values for $n_{j}$, namely $\left(\begin{array}{c}0 \\ \pm 1\end{array}\right)$. Under the action of $\left(A^{*}\right)^{k}$, which involves rotation through $k \pi / 4$, there are only 8 directions $u$ for which $x \cdot u$ does not have a unique maximum, hence we conclude that $K$ is an octagon.

Since there are very few integer similarity matrices with the property that some power is a multiple of the identity, we can say that in the "generic" case $K$ is not a polygon. Our goal is to give a complete description of $K$ in that case. For this discussion we will make the following generic assumption: all directions $\left(A^{*}\right)^{k} n_{j}$ are distinct. Most of what we say can be modified so as to be true in general. 
For each choice of $j$ and $k$, let $u_{j, k}$ denote the unit vector in the direction of $\left(A^{*}\right)^{k} n_{j}$. Then $x \cdot u_{j, k}$ achieves its maximum at 2 distinct points. Indeed, $\left(A^{-k^{\prime}} d\right) \cdot u_{j, k}$ will have a unique maximizer for every $k^{\prime} \neq k$ by the generic assumption, and $\left(A^{-k} d\right) \cdot u_{j, k}$ achieves its maximum for the 2 digits that determine the edge $e_{j}$ of $P$ associated to the normal $n_{j}$. Thus the boundary of $K$ contains a line segment $S_{j, k}$ that is a translate of $A^{-k} e_{j}$. (In the nongeneric case some of these line segments may combine to make longer segments.) If $A$ is a similarity with expansion constant $r$, then the length of $S_{j, k}$ is exactly $r^{-k}\left|e_{j}\right|$, so the sum of the lengths of all the segments is $\frac{1}{r-1}$ times the perimeter of $P$. This gives the lower bound

$$
\operatorname{perimeter}(K) \geq \frac{1}{r-1} \operatorname{perimeter}(P) \text {. }
$$

In Part II [KLSW] we will show that this is in fact an equality.

Another interesting observation is that, under the generic assumption, the boundary of $K$ is a $C^{1}$ curve if $A$ is a similarity. Of course the boundary of a convex set in the plane is differentiable almost everywhere, and can fail to have a derivative only at a "corner point" where left and right derivatives exist but fail to be equal. The sum of the angular jumps (the exterior angles of the corners) at all such corner points can add up to at most $2 \pi$. But in our situation the existence of one such corner point would imply the existence of infinitely many distinct corner points with the same angular jumps, namely the pre-images under the IFS, which are all distinct under the generic assumption. The absence of corner points then implies the boundary in $C^{1}$. But it is easy to see that it cannot be $C^{2}$, because if it were, then a continuous curvature could be defined, and since the curvature is zero on a dense set of points (the line segments), we obtain a contradiction.

We turn now to the case of general $n$. We may assume without loss of generality that $P$ is a nondegenerate polytope, by passing if necessary to an iterate of the original IFS. (If no iterate yields a nondegenerate polytope, then $T$ lies in a lower dimensional subspace.) Let $n_{j}$ denote the outward unit normal vectors to the $n-1$ dimensional faces $f_{j}$ of $P$. Then we have the analog of Theorem 4.1 holding.

Theorem 4.2. Let $T$ be a nonempty compact set satisfying $A(T)=T+\mathcal{D}$ for an expanding matrix $A \in M_{n}(\mathbb{R})$ and a finite digit set $\mathcal{D} \subset \mathbb{R}^{n}$. Let $\left\{n_{j}\right\}$ be the outward unit normal vectors of the $(n-1)$-dimensional faces of the convex hull $P$ of $\mathcal{D}$. Then the convex hull $K$ of $T$ is a polytope if and only if every $n_{j}$ is an eigenvector of $\left(A^{*}\right)^{k}$ for some $k$.

Proof. The necessity of the condition is proved as before, because if there are an infinite number of distinct directions for $\left(A^{*}\right)^{k} n_{j}$ then there will be an infinite number of $n-1$ dimensional faces in $K$ with distinct normal vectors, and so $K$ will not be a polytope. 
Suppose conversely that the eigenvector condition holds. We may assume that the same value of $k$ works for all $j$. Since $P$ is nondegenerate it follows that $\left(A^{*}\right)^{k}$ has a complete set of eigenvectors. Thus we can decompose $\mathbb{R}^{n}$ into a product of subspaces on which $\left(A^{*}\right)^{k}$ acts as a distinct multiple of the identity, say

$$
\mathbb{R}^{n}=\mathbb{R}^{r_{1}} \times \mathbb{R}^{r_{2}} \times \cdots \times \mathbb{R}^{r_{m}} .
$$

Our hypothesis is that each $n_{j}$ belongs to one of these subspaces. We will show in Lemma 4.3 below that this implies that $P$ has the form of a product $P=P_{1} \times P_{2} \times \cdots \times P_{m}$ where each $P_{j}$ is a convex polytope in the $\mathbb{R}^{r_{j}}$ subspace. Since $\left(A^{*}\right)^{k}$ acts as a distinct multiple of the identity on each subspace, it follows that $A^{*}$ preserves each subspace (since $A^{*}$ commutes with $\left(A^{*}\right)^{k}$ ), and so $K$ itself factors into a product $K=K_{1} \times K_{2} \times \cdots \times K_{m}$. But $K_{j}$ is a polytope because a power of $A$ is a multiple of the identity on $\mathbb{R}^{r_{j}}$. So $K$, being the product of polytopes, is itself a polytope.

The following proof of the lemma was suggested to us by Robert Connelly.

Lemma 4.3. Let $P$ be a nondegenerate convex polytope in $\mathbb{R}^{n}=\mathbb{R}^{r_{1}} \times \cdots \times$ $\mathbb{R}^{r_{m}}$ such that each of the normals to the $n-1$ dimensional faces of $P$ lies in one of the subspaces $\mathbb{R}^{r_{j}}$. Then $P$ factors into a product of convex polytopes $P=P_{1} \times P_{2} \times \cdots \times P_{m}$ lying in the given subspaces.

Proof. $P$ is the intersection of half-spaces whose boundaries contain the $n-1$ dimensional faces of $P$. Segregate these half-spaces into $m$ sets according to which of the subspaces the normal to the face belongs to. Thus $P=\widetilde{P}_{1} \cap \widetilde{P}_{2} \cap$ $\cdots \cap \widetilde{P}_{m}$ where each $\widetilde{P}_{j}$ is the intersection of half-spaces with normals lying in the subspace $\mathbb{R}^{r_{j}}$. Thus $\widetilde{P}_{j}$ is a convex set that is the product of a convex set $P_{j}$ in $\mathbb{R}^{r_{j}}$ with all the other subspaces. It follows that $P=P_{1} \times \cdots \times P_{2}$, and since $P$ is a polytope, each $P_{j}$ must also be a polytope.

A description of $K$ in the case when it is not a polytope becomes considerably more difficult in higher dimensions. To illustrate some of the difficulties we consider $n=3$. For each face $f_{j}$ of $P$ and each $k \geq 1$, the boundary of $K$ will contain a translate of $A^{-k} f_{j}$, namely the maximizer of $x \cdot\left(A^{*}\right)^{k} n_{j}$, where $n_{j}$ is the normal of $f_{j}$. However, these will not be the only faces contained in the boundary of $K$. There will also be a family of parallelograms that arise from maximizing $x \cdot u$ when there is a tie for the maximum of $d \cdot\left(A^{-k}\right)^{*} u$ for 2 distinct values of $k$. This possibility cannot be eliminated by a generic assumption. Consider any pair of edges $e_{j m}=f_{j} \cap f_{m}$ and $e_{j^{\prime} m^{\prime}}=f_{j^{\prime}} \cap f_{m^{\prime}}$ of $P$, and any choice of distinct $k$ and $k^{\prime}$. Then the cross product of $A^{-k} e_{j m}$ and $A^{-k^{\prime}} e_{j^{\prime} m^{\prime}}$ (regarded as vectors in $\mathbb{R}^{3}$ ) is a candidate for $u$ (after normalization), since $d \cdot\left(A^{-k}\right)^{*} u$ will produce the same value for the digits at the ends of the edges $e_{j m}$, and similarly for $d \cdot\left(A^{-k^{\prime}}\right)^{*} u$ and the edge $e_{j^{\prime} m^{\prime}}$. However it still has to be checked 
that these ties occur at the maximum values as $d$ varies over $\mathcal{D}_{0}$. Another way of saying this is that the vector $u$, which by construction lies in the intersection of the planes $P_{j \ell}^{k}$ and $P_{j^{\prime} \ell^{\prime}}^{k^{\prime}}$ determined by $A^{-k} n_{j}$ and $A^{-k} n_{\ell}$, and by $A^{-k^{\prime}} n_{j^{\prime}}$ and $A^{-k^{\prime}} n_{\ell^{\prime}}$, must in fact lie in between $A^{-k} n_{j}$ and $A^{-k} n_{\ell}$ in $P_{j \ell}^{k}$, and in between $A^{-k^{\prime}} n_{j^{\prime}}$ and $A^{-k^{\prime}} n_{\ell^{\prime}}$ in $P_{j^{\prime} \ell^{\prime}}^{k^{\prime}}$. There does not appear to be any simple method to decide when this occurs. When it does, it produces a parallelogram of area $\left\|A^{-k} e_{j \ell} \times A^{-k^{\prime}} e_{j^{\prime} \ell^{\prime}}\right\|$ in the boundary of $K$.

Even in the case when $A^{N}$ is a multiple of the identity, hence $K$ is a polytope, it is not clear how to estimate the number of faces of $K$.

\section{APPENDIX}

Let $T$ be a self-affine tile satisfying (1.1) such that $A$ is an expanding integer matrix and $\mathcal{D} \subset \mathbb{Z}^{n}$. In this appendix we describe a simple algorithm that finds the lattice points $\alpha$ such that $T \cap(T+\alpha) \neq \varnothing$.

Since we are concerned with the boundary of $T$, we shall assume that $T$ tiles $\mathbb{R}^{n}$ by $\mathbb{Z}^{n}$-translations, although our algorithm works just fine without this assumption. Then the points on the boundary of $T$ are precisely those in the intersections $T_{\alpha}=T \cap(T+\alpha)$ as $\alpha$ varies over $\mathbb{Z}^{n}$. Our goal is to identify those lattice points $\alpha$ for which $T_{\alpha}$ is nonempty. More generally, for any finite set $F \subseteq \mathbb{Z}^{n}$ we define

$$
T_{F}=\bigcap_{\alpha \in F}(T+\alpha) .
$$

We will assume, without loss of generality, that $F$ contains 0 , since translating the set $F$ also translates $T_{F}$, and that $\#(F) \geq 2$. We let $\mathcal{F}^{*}$ denote the set of sets $F$ containing 0 for which $T_{F}$ is nonempty, and $\mathcal{F}_{m}$ the sets of cardinality $m$ in $\mathcal{F}$. We will describe an algorithm for determining $\mathcal{F}^{*}$.

Lemma A.1. $F$ is in $\mathcal{F}^{*}$ if and only if there exist sequences $d_{j}^{\alpha}$ in $\mathcal{D}$, $j=1,2, \ldots$ for each $\alpha \in F$, such that

$$
\alpha=\sum_{j=1}^{\infty} A^{-j}\left(d_{j}^{0}-d_{j}^{\alpha}\right) .
$$

In the case that $F \in \mathcal{F}^{*}$,

$$
x=\sum_{j=1}^{\infty} A^{-j} d_{j}^{0}
$$

is a point in $T_{F}$. 
Proof. If $T_{F}$ is nonempty and $x \in T_{F}$, we can write $x$ in the form (A.2), and also, since $x-\alpha \in T$, we have

$$
x-\alpha=\sum_{j=1}^{\infty} A^{-j} d_{j}^{\alpha}
$$

for some $d_{j}^{\alpha}$ in $\mathcal{D}$. Subtracting (A.3) from (A.2) we obtain (A.1). The argument also runs in reverse.

Now for any map $\sigma: F \rightarrow \mathcal{D}$ we define $F_{\sigma}=\{A \alpha+\sigma(\alpha)-\sigma(0): \alpha \in$ $F$ \}. Note that $F_{\sigma}$ contains 0 since $0=\sigma(0)$, and $\# F_{\sigma}=\# F$, because if $A \alpha+\sigma(\alpha)-\sigma(0)=A \beta+\sigma(\beta)-\sigma(0)$ then we must have $\alpha=\beta$ because $\mathcal{D}$ is a complete set of residues.

Lemma A.2. $F \in \mathcal{F}^{*}$ if and only if there exists $\sigma: F \rightarrow \mathcal{D}$ such that $F_{\sigma} \in \mathcal{F}^{*}$. Furthermore, every $x$ in $T_{F}$ has the representation (A.2) where

$$
d_{j}^{0}=\sigma_{j} \sigma_{j-1} \cdots \sigma_{1}(0)
$$

for $\sigma_{j}: F_{j-1} \rightarrow \mathcal{D}, F_{j}=\left(F_{j-1}\right)_{\sigma_{j}}, F_{0}=F$, and all $F_{j} \in \mathcal{F}^{*}$.

Proof. If $F$ is in $\mathcal{F}^{*}$, choose $d_{j}^{\alpha}$ according to Lemma A.1, and set $\sigma(\alpha)=d_{1}^{\alpha}$. Then multiply (A.1) by $A$ to obtain

$$
A \alpha+\sigma(\alpha)-\sigma(0)=\sum_{j=1}^{\infty} A^{-j}\left(d_{j+1}^{0}-d_{j+1}^{\alpha}\right) .
$$

It follows by Lemma A.1 that $F_{\sigma} \in \mathcal{F}^{*}$. This argument also works in reverse. For $F$ in $\mathcal{F}^{*}$ we may iterate the result just obtained to get the sequence $F_{0}, F_{1}, \ldots$ in $\mathcal{F}^{*}$ and the maps $\sigma_{j}$, and from (A.2) we obtain (A.4).

We can summarize the above lemma by a graph $G$ (a directed multigraph, which allows multiple edges joining vertices, and edges joining a vertex to itself). The points in $G$ are the sets $F$ in $\mathcal{F}^{*}$, and the directed edges are the maps $\sigma$ for which $F_{\sigma}$ is also in $\mathcal{F}^{*}$ (the directed edge $\sigma$ goes from $F$ to $F_{\sigma}$ ). Points in $T_{F}$ correspond to infinite paths in $G$ starting at the vertex $F$. (There is not necessarily a one-to-one correspondence, because points may have more than one representation (A.2).) The lemma says that every path in $G$ is indefinitely extendable. We could also restrict attention to sets of fixed cardinality, the resulting graphs being denoted $G_{m}$.

Now we can describe a simple pruning algorithm to find the graph $G_{m}$. We start with a finite graph $G_{m}^{(1)}$ that contains $G_{m}$. From (1.2) we have an a priori bound for the diameter of $T$, and this gives an a priori bound for the diameter of any set in $\mathcal{F}_{m}$ (this also gives an a priori bound for $m$ such that $\mathcal{F}_{m}$ is nonempty). 
Thus we may take $G_{m}^{(1)}$ to have vertices all sets $F$ containing 0 and of limited diameter, and take all $\sigma$ with the property that $F_{\sigma}$ is also a vertex in $G_{m}^{(1)}$.

The pruning algorithm to go from $G_{m}^{(j)}$ to $G_{m}^{(j+1)}$ consists of removing all vertices with no edges going out, and then all edges going into the removed vertices. After a finite number of steps the pruning algorithm stops because all vertices have outgoing edges.

Theorem A.3. $\mathcal{F}_{m}$ is exactly the set of vertices of $G_{m}$ when the pruning algorithm stops.

Proof. It is clear from Lemma A.2 that the pruning algorithm never removes sets in $\mathcal{F}_{m}$. So we need to show that every vertex in $G_{m}$ is a set in $\mathcal{F}_{m}$, or that $T_{F}$ is nonempty for such sets $F$. Since $G_{m}$ is by construction a graph with every path infinitely extendable, we can construct an infinite path in $G_{m}$ starting at $F$, and thus a sequence of maps $\sigma_{j}: F_{j-1} \rightarrow \mathcal{D}$ with $F_{j}=\left(F_{j-1}\right)_{\sigma_{j}}$. We take $d_{j}^{\alpha}=\sigma_{j} \sigma_{j-1} \cdots \sigma_{1}(\alpha)$. We want to show that (A.1) holds, for then we will have $F \in \mathcal{F}_{m}$ by Lemma A.1. What we know is (by induction) that

$$
A^{k+1} \alpha+A^{k}\left(d_{1}^{\alpha}-d_{1}^{0}\right)+A^{k-1}\left(d_{2}^{\alpha}-d_{2}^{0}\right)+\cdots+A\left(d_{k}^{\alpha}-d_{k}^{0}\right)
$$

is a point in one of the sets in $G_{m}$, which all have bounded diameter. Multiplying by $A^{-k-1}$ we obtain

$$
\alpha-\sum_{j=1}^{k} A^{-j}\left(d_{j}^{0}-d_{j}^{\alpha}\right)=A^{-k-1} z_{k}
$$

where $z_{k}$ are uniformly bounded. We can then let $k \rightarrow \infty$ to obtain (A.1).

Remark. Note that the set $\mathcal{F}$ we have used in $\S 2$ is simply the set $\mathcal{F}_{m}$ here for $m=2$.

\section{REFERENCES}

[B] C. BANDT, Self-similar sets 5. Integer matrices and fractal tilings of $\mathbb{R}^{n}$, Proc. Amer. Math. Soc. 112 (1991), 549-562.

[DK] P. Duvall AND J. KeEsling, The Hausdorff dimension of the boundary of the Levy dragon, Int. J. Math. and Math. Sci. 20 (1997), 627-632.

[DKV] P. Duvall, J. Keesling And A. Vince, The Hausdorff dimension of the boundary of a self-similar tile, preprint.

[Fa] K. FAlCONER, Techniques in fractal geometry, John Wiley \& Sons, (1997).

[FW] T. Flaherty AND Y. WANG, Haar-type multiwavelet bases and self-affine multi-tiles, Asian J. Math. 3 (1999), 387-400.

[GH] K. GröChEnIG AND A. HAAs, Self-similar lattice tilings, J. Fourier Analysis and Appl. 1 (1994), 131-170.

[K1] R. KenYon, Self-replicating tiling, Contemp. Math. 135 (1992), 239-263

[KLSW] R. Kenyon, J. Li, R. Strichartz And Y. WAng, Geometry of self-affine tiles II, Indiana Univ. Math. J. 48 (1999), 25-42. 
[LW1] J. Lagarias AND Y. WANG, Integral self-affine tiles in $\mathbb{R}^{n}$ I. Standard and nonstandard digit sets, J. London Math. Soc. 53 (1996) 21-49.

[LW2] J. Lagarias ANd Y. WANG, Self-affine tiles in $\mathbb{R}^{n}$, Adv. in Math. 121 (1996), 21-49.

[LW3] J. LAGARIAS AND Y. WANG, Integral self-affine tiles in $\mathbb{R}^{n}$ Part II: Lattice tilings, J. Fourier Anal. Appl. 3 (1997), 84-102.

[MW] D. Mauldin AND S. Williams, Hausdorff dimension in graph directed constructions, Trans. Amer. Math. Soc. 309 (1988), 811-829.

[Ve] J. VeERman, Hausdorff dimension of boundaries of self-affine tiles in $\mathbb{R}^{n}$, preprint.

Robert S. Strichartz is supported in part by the National Science Foundation, grant DMS-9623250. Yang Wang is supported in part by the National Science Foundation, grant DMS-9706793.

Robert S. STRICHARTZ

Mathematics Department

Malott Hall

Cornell University

Ithaca, New York 14853

EMAIL: str@math.cornell.edu

YANG WANG

School of Mathematics

Georgia Institute of Technology

Atlanta, Georgia 30332

EMAIL: wang@math.gatech.edu

Received: April 16th, 1998; revised: December 9th, 1998. 